\title{
LIDERAZGO COMO FACTOR CRÍTICO \\ DE ÉXITO PARA LA GESTIÓN \\ DE LA CALIDAD DE LAS PYMES. \\ REFLEXIONES PARA LA DISCUSIÓN
}

\author{
Roselin Santamaría-Peraza, Agustín Mejías-Acosta* \\ Universidad de Carabobo, Venezuela
}

Recibido: 20 de agosto del 2019 / Aprobado: 12 de marzo del 2020

doi: 10.26439/ing.ind2020.n038.4815

RESUMEN: El objetivo de este estudio es establecer las variables que determinan el liderazgo, como factor crítico para el éxito de la gestión de la calidad. La investigación es de tipo documental con un nivel descriptivo. Como hallazgo principal, se resalta la importancia del liderazgo, en la conducción de los procesos relacionados con la calidad y mejora de esta. Así mismo, se establece que una de las características más resaltantes de un líder es su capacidad de facilitarle a los demás para que actúen y lo sigan, así como su orientación a los resultados.

Palabras clave: liderazgo / gestión de la calidad total / pequeñas y medianas empresas

\section{LEADERSHIP AS A CRITICAL SUCCESS FACTOR \\ FOR SMALL AND MEDIUM ENTERPRISES' QUALITY MANAGEMENT. REFLECTIONS FOR DISCUSSION}

\begin{abstract}
The objective of this study is to establish the variables that determine leadership as a critical factor for quality management success. The research was conducted as a documentary analysis with a descriptive approach. The main finding of the research was the importance of leadership to carry out processes related to its quality and improvement. Moreover, it is concluded that the most prominent skills of leaders are their ability to influence others to act, their ability to be followed, and their willingness to achieve results.
\end{abstract}

Keywords: leadership / total quality management / small and medium enterprises

\footnotetext{
*roselin2602@gmail.com, amejiasa@uc.edu.ve
} 


\section{INTRODUCCIÓN}

La globalización de los mercados es un elemento de la nueva economía, en ella existe un intercambio entre los mercados, tanto nacionales como internacionales, implicando esto una mayor competencia, requiriendo estrategias que permitan a las empresas garantizar su participación en el mercado para ser la primera opción de los consumidores. Por ello las organizaciones constantemente se encuentran mejorando y haciéndose más eficaces para certificar su persistencia en el entorno donde se desarrollan, debido a que los requerimientos de los mercados cambian en la búsqueda de la satisfacción de clientes cada vez más exigentes.

El sector empresarial está conformado por grandes, medianas y pequeñas empresas, siendo, de acuerdo con Franco y Urbano (2010), las pymes las organizaciones que generan mayor cantidad de empleos, en algunos casos hasta un 73 \%; así mismo, se puede señalar que estas representan, aproximadamente, el $99 \%$ del total de compañías, contribuyendo así con el $30 \%$ del producto interno bruto (PIB) en América Latina y con el 60 \% en Europa, de acuerdo con lo expuesto por la Comisión Económica para América Latina y el Caribe (CEPAL, 2012) y la Organización para la Cooperación y Desarrollo Económicos (OCDE), respectivamente. Por otra parte, la OCDE en conjunto con el Banco de Desarrollo de América Latina (OCDE y CAF, 2019), recientemente, señalan que más del $99,5 \%$ de las empresas en América Latina y el Caribe son pymes, las cuales generan el $60 \%$ del empleo productivo formal, convirtiéndose en motores críticos para el crecimiento y la inclusión social a nivel regional. Sin embargo, estas empresas, a nivel de Latinoamérica, se enfrentan a la competencia del sector informal y a una brecha de productividad significativa respecto a las grandes empresas; de ahí la importancia de realizar estudios que les permitan a las pymes mejorar dentro del contexto productivo, así como potenciarlas para ser agentes de cambio estructural del tejido empresarial.

Debido a la importancia de las pymes para el desarrollo de las comunidades donde se encuentran, estas deben buscar mecanismos para su desarrollo, orientando sus acciones en el análisis de sus factores internos y así contrarrestar a los factores externos, para generar una capacidad estratégica clave que las lleve a una posición diferenciada en el mercado. Por lo que, cuando una empresa genera mecanismos que le permiten mantenerse en función del tiempo, se considera que es exitosa y sostenible (Arrieta et al., 2014).

De acuerdo con Wanjiru y Kilika (2016), las competencias son el vínculo entre los recursos internos de la empresa y su entorno; una adecuada gestión de estos factores internos para adaptarse a los cambios y lograr sobresalir hacen que las organizaciones sean consideradas competitivas; es decir, compañías con capacidad para mantener sistemáticamente ventajas competitivas que les permitan alcanzar, sostener y mejorar una determinada posición en el entorno socioeconómico donde se encuentren (Luna, 2012). 
Dos empresas pueden poseer recursos de fuentes comunes, pero la manera como son utilizados para crear procesos, difíciles de imitar, que le permitan sobresalir entre todos, en el desarrollo de ventajas competitivas, es la diferencia del éxito entre una de ellas (Wanjiru y Kilika, 2016). Considerando ello, existen factores que permiten alcanzar ventajas competitivas, tales como, la calidad, la innovación, la capacitación del personal, el enfocarse al cliente, la mejora continua, la gestión del conocimiento, entre otros.

Sin embargo, hay investigadores que apoyan la calidad como fuente de ventaja competitiva, estos son Ramseook-Munhurrun, Munhurrun y Panchoo (2011), Rougan (2015), Hitt, Ireland y Hoskisson (2015), García-Cabrera y García-Soto (2016), entre otros; quienes consideran que la satisfacción al cliente es una de las formas para permanecer en el mercado y sobresalir entre los otros competidores. Al considerar la calidad como fuente de ventaja, es necesario revisar los modelos de gestión, ya que la utilización de estos proporciona a las empresas un marco para el desarrollo de políticas, estrategias y compromisos que les permite alcanzar los objetivos propuestos mediante la satisfacción de los clientes.

La calidad puede abarcar todos los procesos de la empresa, considerando incluso modelos de gestión de la calidad (González, Tapia, Hernández y Luna, 2016), ya que estos poseen un enfoque integrado que busca obtener y mantener, a través de la mejora continua, niveles de producción de calidad, considerando que el mejor sistema es el basado en la filosofía de control total de la calidad, siendo un elemento clave para mantener una ventaja competitiva (Talib, Ali e Idris, 2013).

Considerando lo anterior, el presente artículo realiza un análisis descriptivo de los diferentes modelos y filosofías de la gestión de la calidad con la finalidad de establecer los requisitos de un buen liderazgo que apoye dichos procesos de gestión de la calidad, a partir de una investigación documental.

\section{METODOLOGÍA}

Con la finalidad de alcanzar las metas trazadas del estudio, este se enmarca en la investigación de tipo descriptiva, debido a que mediante esta se busca especificar propiedades y características importantes del liderazgo en la gestión de la calidad. Su desarrollo se realiza como una investigación de tipo documental, debido a que se fundamenta en el análisis de contenido utilizando resúmenes y análisis de la información. Para ello, se tomaron como fuente primaria artículos relacionados, considerando las bases de datos, buscando palabras claves tales como: gestión de la calidad, normas ISO, filosofías de mejora continua, modelos de gestión de la calidad, competitividad, ventaja competitiva, pymes. 
Esta es la parte inicial de un estudio el cual tiene como finalidad establecer los factores críticos de la variable liderazgo.

\section{ANÁLISIS Y DISCUSIÓN}

Un factor importante en el desarrollo de los procesos de la calidad es el liderazgo. Según Díaz Figueroa, Medina Quintero y de la Garza Ramos (2011), el líder influye con su conducción en la forma de tomar riesgos, como se involucra en el futuro de los empleados y teniendo en cuenta las opiniones de sus subalternos. Para Gil-Osorio e Ibarra-Lopesierra (2014), el liderazgo es el arte de influir en la conducta de las personas con la finalidad de lograr los objetivos de las organizaciones, este y los factores críticos del éxito son claves para lograr un impacto significativo en la consecución de objetivos de una organización en los actuales mercados. De acuerdo con el modelo de excelencia europea, un fuerte liderazgo en conjunto con una dirección estratégica clara, se logra el éxito sostenido (Fundación Europea para la Gestión de la Calidad [EFQM], 2018). Leonard (2010) señala que el liderazgo organizacional junto con un núcleo ético, centrado en las personas, la responsabilidad social y la calidad de la vida laboral, son elementos esenciales de la calidad, citando a Deming, Juran y Feigenbaum.

Ayuso y Herrera (2017) señalan que los líderes que son capaces de adaptarse para satisfacer las demandas de cada situación particular tienen mayor capacidad de influenciar en el comportamiento de su equipo. Por otra parte, si se desea mejorar el nivel de madurez de un colaborador, para que este asuma una responsabilidad significativamente mayor, lo mejor que puede hacer un líder es reducir la conducta de tarea y aumentar la conducta de relación, con base en ello se señala que la conducta de relación no solo favorece la percepción de mejor clima laboral, sino además disminuye la percepción de presión y control en el trabajo.

El líder, fundamentado en lo anterior, es la persona que debe orientar o guiar a los demás en el proceso de alcanzar los objetivos. En una organización esa función, en algunos casos, la ejerce el gerente, sin embargo, no necesariamente es quien realmente posee la capacidad de motivar a las personas, pero es quien debe ejercer el liderazgo de la forma más adecuada según las necesidades de la organización.

Se considera como calidad a la traducción de los requerimientos de los clientes en características capaces de ser percibidas por ellos, tales como que el producto cumpla con las especificaciones de diseño, así como que no posea disconformidades, y que los consumidores puedan pagarlo. Esta concepción de calidad ha generado un creciente interés como factor de competitividad, desarrollándose modelos de gestión que tienen como finalidad orientar esfuerzos para obtener servicios o productos con altos estándares, pero que el consumidor pueda pagar (Santamaría Peraza, 2017). 
Para establecer la relación entre liderazgo y calidad, se revisan a continuación algunos modelos de gestión de la calidad, así como filosofías de mejora, los cuales buscan integrar funciones de forma estructurada con el propósito de alcanzar los objetivos. Entre los modelos y filosofías más utilizados se encuentran:

\subsection{Modelo de gestión de la calidad basado en procesos (IS0 9000)}

Este modelo lo conforma un conjunto de normas pertenecientes a la familia ISO 9000, las cuales son un consenso internacional de buenas prácticas de gestión, con el objetivo de que una organización pueda entregar productos y servicios que satisfagan los requisitos de calidad de los clientes. Estas normas son publicadas y mantenidas por la Organización Internacional de Estandarización (ISO, por sus siglas en inglés, International Organization for Standardization). Además, aportan reglas básicas para desarrollar un sistema de calidad, independiente del fin de la empresa o del producto o servicio que proporcione.

Este modelo considera los siguientes principios para la administración de la calidad: enfoque para el cliente, liderazgo, participación de la gente, enfoque hacia el proceso, enfoque de sistemas para la administración, mejora continua, enfoque hacia la toma de decisiones basados en hechos y datos, relación con los proveedores para beneficio mutuo. De acuerdo con Cruz, López y Ruiz (2017), estas normas apoyan al proceso de mejora continua de las organizaciones, permitiendo la optimización de los productos ofrecidos al consumidor final.

\subsection{El modelo del premio Malcolm Baldrige}

El modelo Baldrige, en honor al secretario de comercio de los Estados Unidos de Norteamérica, Malcolm Baldrige, quien fue un defensor de la gestión de calidad como elemento clave para la prosperidad y sostenibilidad del país, nació como objetivo de mejorar la competitividad de las empresas norteamericanas, ampliándose luego hacia la atención sanitaria y a las organizaciones de educación, así como a las organizaciones sin fines de lucro y a las organizaciones gubernamentales. Hoy en día es una guía para mejorar la calidad de los productos y servicios de muchas organizaciones en diferentes países (Schniederjans, Mellat, Nabavi, Subba y Raghu-Nathan, 2018). Su evaluación se basa en la revisión de ciertos criterios, los cuales se denominan criterios de excelencia, que ayudan a las organizaciones a alinear los recursos y mejorar la comunicación, la productividad y la eficacia, con la finalidad de alcanzar los objetivos estratégicos. Estos criterios representan un marco integrado para la gestión y son: el liderazgo, las estrategias, los clientes, la medición, el análisis y el conocimiento gerencial; la fuerza de trabajo; los procesos y resultados. 


\subsection{Modelo del premio de excelencia europeo (EFQM)}

Considerando la revisión EFQM (2018), este se compone de tres elementos: los conceptos fundamentales, el modelo de excelencia EFQM, y un esquema de estructuración basado en la lógica de radar. El modelo se encuentra compuesto de un marco no-prescriptivo basado en nueve criterios y treinta y dos subcriterios, que permiten a los líderes de las organizaciones entender la causa y efecto entre lo que la organización hace y los resultados que logra. De los nueve criterios, cinco son "habilitadores" y cuatro son "resultados". Este modelo es flexible y puede ser aplicado a pequeñas o grandes organizaciones tanto del sector público como del privado, bajo la premisa: los resultados excelentes con respecto al rendimiento de la organización, a los clientes, el capital humano y la sociedad se logran a través de un liderazgo que dirija e impulse la política y estrategia, las personas de la organización, las alianzas, los recursos y los procesos (Esquivel, León y Castellanos, 2017).

\subsection{Modelo iberoamericano de excelencia}

Es la base utilizada para la evaluación de las organizaciones candidatas a los premios a la calidad que entrega la Fundación Iberoamericana para la Gestión de la Calidad (FUNDIBEQ). Se estructura en 5 procesos facilitadores: 1) liderazgo y estilo de gestión, 2) estrategia, 3) desarrollo de las personas, 4) recursos y asociados, y 5) procesos y clientes. Cada uno se halla dividido, para su análisis, en cuatro subcriterios con puntajes definidos que ayudan a la puntuación final deducida de la valoración.

\subsection{Modelo de calidad de México}

Este modelo se llama modelo nacional para la competitividad (MCN) y busca impulsar la mejora continua de las organizaciones mexicanas sin importar su tamaño para proyectarlas, de forma ordenada, a niveles de competitividad de clase mundial. Promueve la utilización de prácticas o herramientas de acuerdo con las características de cada organización y su entorno, sin considerar una teoría o corriente específica. Se compone de 8 criterios, que a la vez se dividen en subcriterios, los cuales representan los elementos fundamentales del sistema organizativo. Los valores que sustentan el modelo son: 1) enfoque al cliente, 2) liderazgo efectivo / ejemplar, 3) personal comprometido, 4) compromiso con la sociedad, 5) mejora continua e innovación y 6) pensamiento sistémico (Palacios Blanco, 2006).

\subsection{Lean manufacturing}

El lean manufacturing es una filosofía de gestión que busca la eliminación de los desperdicios, incluyendo todas aquellas actividades que no aportan valor al producto y por las cuales el cliente no está dispuesto a pagar, esto mediante la aplicación de diferentes 
herramientas administrativas y de producción que incluye, entre otras, la mejora continua (León, Marulanda y González, 2017). De acuerdo con Alefari, Salonitis y Xu, (2017), el liderazgo y el compromiso de la alta gerencia son factores claves en la implementación de manufactura esbelta o lean manufacturing, debido a que este amerita un cambio en la conducción de las organizaciones y se debe tener una claridad para fijar la ruta de acción para alcanzar el éxito en la implementación.

El liderazgo está ligado a las actividades de dirección y gestión, el desarrollo de este es un elemento fundamental en los avances de la gestión de la calidad total en las organizaciones (Ayuso y Herrera, 2017) y se define considerando las características y cualidades del líder (Leonard, 2010). Como se puede detallar, en los modelos de calidad anteriores, entre sus criterios o principios, el liderazgo ocupa un lugar importante para la elaboración de procesos; fundamentado en cada modelo y filosofía de mejora, así como en otros investigadores sobre el tópico. En la tabla 1 se pueden observar las diferentes definiciones y características planteadas según los diferentes enfoques citados.

Tabla 1

El liderazgo de acuerdo con diferentes enfoques de gestión de la calidad

\begin{tabular}{|c|c|c|}
\hline Enfoque & Definición & Características \\
\hline ISO 9000 & $\begin{array}{l}\text { Establece la unidad de propósito y la di- } \\
\text { rección; crea condiciones para que las } \\
\text { personas se impliquen en el logro de los } \\
\text { objetivos de calidad de la organización. }\end{array}$ & $\begin{array}{l}\text { Con base a los requerimientos de la } \\
\text { norma, los líderes deben ser comuni- } \\
\text { cadores efectivos, ser ejemplos positi- } \\
\text { vos, e inspirar, fomentar y reconocer la } \\
\text { contribución de las personas. }\end{array}$ \\
\hline EFQM & $\begin{array}{l}\text { Un líder debe estar enfocado en lograr } \\
\text { objetivos a través de las personas. La } \\
\text { mayoría tiene una visión clara y pueden } \\
\text { comunicarla. Es un agente de cambio; } \\
\text { inspira y motiva. Además, suelen ser } \\
\text { modelos para seguir enfocados en la } \\
\text { integridad, la responsabilidad social y } \\
\text { el comportamiento ético, tanto interna } \\
\text { como externamente, asegurando que su } \\
\text { personal adopte los más altos estándares } \\
\text { de comportamiento ético. }\end{array}$ & $\begin{array}{l}\text { El líder EFQM da aliento al corazón, mo- } \\
\text { dela el camino, es capaz de manejar el } \\
\text { cambio, es flexible e inspira confianza. }\end{array}$ \\
\hline Malcom Baldrige & $\begin{array}{l}\text { El líder debe garantizar el desarrollo de } \\
\text { estrategias, sistemas y métodos para } \\
\text { alcanzar el desempeño de excelencia, } \\
\text { estimulando la innovación y constru- } \\
\text { yendo conocimientos y capacidades } \\
\text { organizacionales. }\end{array}$ & $\begin{array}{l}\text { Este líder debe inspirar y motivar a } \\
\text { sus colaboradores; estimularlos a } \\
\text { contribuir, desarrollarse y aprender, a } \\
\text { ser innovadores y creativos. Mantiene } \\
\text { una conducta ética, planificando sus } \\
\text { funciones en la comunicación y en el } \\
\text { coaching. }\end{array}$ \\
\hline
\end{tabular}


(continuación)

FUNDIBEQ Desarrolla y pone en práctica la cultura y los valores de la organización, mediante adecuados comportamientos y acciones éticas; así como la estructura organizativa y el marco de los procesos necesarios para la eficaz ejecución de la política y la estrategia.

Modelo de calidad Los líderes son los impulsores del camnacional de México bio, son los que deben convertir el decir (Palacios Blanco, 2006) en el hacer, así como ser responsables de crear una cultura de calidad y establecer su rumbo estratégico.

Líderes que inspiran confianza; son flexibles, deben ser modelo de integridad, responsabilidad social y comportamiento ético; ser activos, estimular la mejora, la innovación y la creatividad; ser accesibles, saben escuchar y dan respuestas.

Líderes que establecen directrices y crean visión centrada en el cliente; enseñan con el ejemplo a tener un comportamiento ético y congruente para involucrar, comunicar, dar asistencia y apoyo, reconocer el desempeño de sus colaboradores, poseer una visión de largo plazo y un pensamiento sistémico.

\begin{tabular}{lll}
\hline Lean manufacturing & El liderazgo juega un papel importante & Los líderes deben centrarse en el uso \\
& para implantar lean, ya que este debe & eficiente de los recursos; ser ejemplo \\
guiar a la organización para el proceso de & a seguir, necesitan ser consistentes a \\
implementación (Alefari et al., 2017; León & largo plazo, basados en la filosofía y \\
et al., 2017). & valores lean (Alefari et al., 2017). Deben \\
& poseer experiencia en implementación \\
& de lean (León et al., 2017).
\end{tabular}

Elaboración propia

Así mismo, se entiende al liderazgo como la función que ejercen las personas en búsqueda del desarrollo y alcance de los objetivos de la organización, de dirigir, ordenar y consolidar el esfuerzo colectivo hacia la continua innovación y adaptación a los cambios del ambiente empresarial, "liderar es un arte, pero hay que desarrollarlo, pues no es suficiente ejercerlo con el instinto o carisma que se posea para ello" (Enríquez Castillo y López Ávila, 2016; Guerrero, 2017). Mediante un fuerte liderazgo en conjunto con una dirección estratégica clara, se logra el éxito sostenido (EFQM, 2018).

De acuerdo con Aguirre León, Serrano Orellana y Sotomayor Pereira (2017), los elementos influyentes para que una empresa logre la perdurabilidad, la calidad y el éxito en el mercado son el resultado del comportamiento y la actitud del gerente; fundamentado en ello, se establece que para generar cambios, mejorar los procesos, lograr que las personas se motiven a lograr los resultados en las organizaciones, se debe contar con gerentes que inicien, faciliten, gestionen, impulsen y coordinen el proceso de transformación. Lo anterior también lo refiere Guerrero (2017), quien señala a los líderes empresariales como aquellos que orientan a la organización dentro de los lineamientos que les permitan alcanzar los objetivos planteados. 
Fundamentado en otros estudios, Ayuso y Herrera (2017) presentan los siguientes estilos de liderazgo: autocrítico, participativo, democrático y liberal, así como los tipos transformador y transaccional. Con relación a los estilos, cada líder toma la decisión del estilo adoptado y esto dependerá de la situación a que se enfrente. Es importante destacar que un elemento importante en la efectividad del trabajo es la flexibilidad, por lo que se debe tener un conocimiento, capacidad y disposición para escoger el estilo de liderazgo; así como establecer la capacidad de relacionarse con el equipo, conocer, escuchar y comunicarse con este de forma activa, con el objetivo de alcanzar el máximo conocimiento posible en el ámbito laboral de cada uno de los colaboradores que forman parte del equipo.

Por otra parte, Enríquez Castillo y López Ávila (2016) señalan al empresario exitoso como una persona con las siguientes características: debe ser un líder, brillante y seguro de sí mismo, reconocer sus deficiencias y cualidades, ser flexible, sensato, convincente y tolerante con los demás; debe saber cuándo y cómo actuar y afrontar las consecuencias de sus decisiones; ser observador y estar atento a lo que sucede; su experiencia y sus conocimientos son reconocidos; ser carismático, expresar correctamente sus ideas, aclarando las dudas que puedan surgir, ser amable y tener un perfil multicultural. Su liderazgo lo ejerce a través de la motivación y el convencimiento, indicando el rumbo a seguir en el presente y mirando al futuro como la oportunidad de orientar creativa, innovadora y positivamente a sus colaboradores y a la empresa en su conjunto.

Para Fernández (2017), el papel del líder es lograr los objetivos a partir del trabajo en equipo, para ello debe apoyarse en su equipo, saber delegar, empoderar, compartir y ceder su posición de líder cuando se requiera. Debido a la complejidad del mercado actual, el líder necesita tener ciertas características como poseer pensamiento global, entender y respetar las diferencias culturales, estar familiarizado con los desarrollos de las tecnologías de la información y comunicación, tener la capacidad de conformar redes y alianzas que permitan complementar cualquier deficiencia del equipo, mostrar un comportamiento ético y, especialmente, saber compartir el poder.

Considerando las revisiones realizadas, en la tabla 2 se detallan las características del líder o persona que ejerce el liderazgo. 
Tabla 2

Características relacionadas con el liderazgo

\begin{tabular}{ll}
\hline \multicolumn{1}{c}{ Características } & \multicolumn{1}{c}{ Investigador } \\
\hline 1. Dar aliento al corazón & Spears (2010), Díaz Figueroa et al. (2011), Pedraza, Lavín, Delgado, \\
& y Bernal (2014), EFQM (2018), Aguirre León et al. (2017) \\
\hline 2. Modelar el camino & Díaz Figueroa et al. (2011), Bonifaz Villar (2012), Pedraza et al. \\
& (2014), Montañez Moya (2015), Aguirre León et al. (2017), Lezama, \\
& Cruz y Pico (2015), EFQM (2018), Fernández (2017) \\
\hline
\end{tabular}

3. Habilitar a los demás para que Sistema Económico Latinoamericano y del Caribe ([SELA], 2010), actúen / para que lo sigan Spears (2010), Díaz Figueroa et al. (2011), Quintero, Álvarez, Calderón y Granada (2011), Bonifaz Villar (2012), López Martínez (2013), Pedraza et al. (2014), Lezama et al. (2015), Aguirre León et al. (2017), Fernández (2017)

4. Inspirar una visión compartida Díaz Figueroa et al. (2011), Pedraza et al. (2014), Aguirre León et al. (2017)

5. Desafiar los procesos Díaz Figueroa et al. (2011), Pedraza et al. (2014), Lezama et al. (2015), Aguirre León et al. (2017)

\begin{tabular}{lll}
\hline 6. Ser visionario & $\begin{array}{l}\text { Spears (2010), López Martínez (2013), Lezama et al. (2015), } \\
\text { Montañez Moya (2015), Aguirre León et al. (2017) }\end{array}$ \\
\hline 7. Ser un comunicador efectivo & Spears (2010), SELA (2010), Bonifaz Villar (2012) \\
\hline 8. Estar enfocado a la orientación a & SELA (2010), Bonifaz Villar (2012), López Martínez (2013), \\
& los resultados & Montañez Moya (2015), Lezama et al. (2015), Fernández (2017) \\
\hline 9. Trabajar en equipo & SELA (2010), Spears (2010), Quintero et al. (2011), Bonifaz Villar \\
& (2012) López Martínez (2013), Lezama et al. (2015), Fernández \\
& (2017), Aguirre León et al. (2017) \\
\hline
\end{tabular}

10. Capaz de manejar el cambio / ser Bonifaz Villar (2012), López Martínez (2013), Lezama et al. (2015), flexible Enríquez Castillo y López Ávila (2016), Guerrero (2017), Fernández (2017), EFQM (2018)

\begin{tabular}{|c|c|}
\hline $\begin{array}{l}\text { 11. Tener perseverancia, persistencia } \\
\text { para lograr el éxito }\end{array}$ & Bonifaz Villar (2012), Enríquez Castillo y López Ávila (2016) \\
\hline 12. Ser analítico & $\begin{array}{l}\text { Bonifaz Villar (2012), López Martínez (2013), Enríquez Castillo y } \\
\text { López Ávila (2016) }\end{array}$ \\
\hline 13. Tener buena atención al cliente & Lezama et al. (2015), Enríquez Castillo y López Ávila (2016) \\
\hline $\begin{array}{l}\text { 14. Ser positivo, no dejarse caer ante } \\
\text { resultados negativos o no desea- } \\
\text { dos o esperados }\end{array}$ & Bonifaz Villar (2012), Enríquez Castillo y López Ávila (2016) \\
\hline 15. Asumir riesgos & Aguirre León et al. (2017) \\
\hline $\begin{array}{l}\text { 16. Reconocer de manera pública } \\
\text { las contribuciones individuales y } \\
\text { celebrar los logros del equipo }\end{array}$ & Aguirre León et al. (2017), Guerrero (2017) \\
\hline $\begin{array}{l}\text { 17. Buscar adquirir y capitalizar el } \\
\text { conocimiento }\end{array}$ & Spears (2010), Lezama et al. (2015) \\
\hline $\begin{array}{l}\text { 18. Saber determinar la importancia } \\
\text { de la integridad de las acciones }\end{array}$ & Lezama et al. (2015) \\
\hline
\end{tabular}

Elaboración propia 
Por otro lado, Pertúz (2018) considera que el liderazgo transformacional permite motivar a sus trabajadores, transmitir las ideas de colaboración, fomentando un ambiente de compromiso, respeto, tolerancia, diálogo, responsabilidad, entre otros, en un contexto dinámico, como el actual, este tipo de liderazgo puede adaptarse a los cambios donde se persigue el trabajo en equipo. Así mismo, Ayuso y Herrera (2017) señalan que este tipo de liderazgo apoya a organizaciones cuyos objetivos son fomentar la creatividad y la innovación entre sus empleados, con la finalidad de mejorar o mantener su posición competitiva.

Todas las características y definiciones señaladas son las que deben tener los líderes para que puedan, entre sus colaboradores, alcanzar los objetivos trazados. Estas son combinaciones que deben ser consideradas y tomadas como punto de partida para establecer cuáles serían las deseables.

\section{RESULTADOS}

El liderazgo es la función que ejerce el líder con la finalidad de alcanzar los objetivos, por lo cual es el encargado de motivar, orientar y dirigir a las personas responsables del logro de estos. Dentro de las pymes, las funciones de liderazgo las ejerce, en muchas ocasiones, el gerente encargado o el dueño de la organización, el cual posiblemente no posee habilidades de liderazgo, siendo necesario desarrollarlas para lograr sus metas. Por ello, debe ser capaz de orientar y dirigir a su personal para alcanzar los objetivos, así como tener la habilidad de ser multidimensional, puesto que en algunos casos debe establecer planes a mediano y largo plazo y, además, formar equipos de trabajo para cumplir estos planes con acciones de corto plazo.

Como se detectó, en los sistemas de gestión de la calidad, el liderazgo es un factor importante porque la conducta, como su capacidad de tomar decisiones, le permitirán establecer diferentes alternativas de acción, tales como desarrollar procesos para lograr las metas y solventar las situaciones problemáticas que se presenten.

Tanto las filosofías de calidad como los modelos de gestión poseen estructuras o rutas de acción que permiten su establecimiento y poder "ser" adaptados a las organizaciones, sean de manufactura o servicio, sin importar el tamaño de las mismas, tomando como requisito importante, para su establecimiento, el contar con el apoyo de la alta gerencia o junta directiva.

Para el caso de las pymes, es necesario establecer de forma objetiva, cuáles de las características mencionadas son las de mayor importancia y las mínimas necesarias que deben tener estos líderes encargados del desarrollo de estas empresas. Esto con la finalidad de ayudarlas a alcanzar sus objetivos al corto, mediano y largo plazo, permitiéndoles permanecer en el sector o mercado donde se encuentren. 
Finalmente, se resaltan las siguientes características que debe tener un líder: modelar el camino, con su conducta y trabajo ético, ser un comunicador efectivo, dar aliento de corazón, habilitar a los demás para que actúen / para que lo sigan, inspirar una visión compartida, desafiar los procesos, ser visionario, generar procesos orientados a los resultados, trabajar en equipo, capaz de manejar el cambio / ser flexible.

\section{REFERENCIAS}

Aguirre León, G., Serrano Orellana, B., y Sotomayor Pereira, G. (2017). El liderazgo de los gerentes de las pymes de Machala. Universidad y Sociedad, 9(1), 187-195. Recuperado de http://rus.ucf.edu.cu/

Alefari, M., Salonitis, K., y Xu, Y. (2017). The role of leadership in implementing lean manufacturing. Procedia CIRP, 63, 756-761. doi:https://doi.org/10.1016/j.procir. 2017.03.169

Arrieta, D., Figueroa, E., Luna, J., Rivera, M., Meléndez, M., y Sotelo, J. (2014). La importancia de la planeación estratégica en la innovación y permanencia de las pymes. Global Conference on Business and Finance Proceedings, 9(2), 378-385. Recuperado de https://www.theibfr.com/wp-content/uploads/2016/06/ISSN1941-9589-V9-N2-2014-1.pdf

Ayuso, D., y Herrera, I. (2017). El liderazgo en los entornos sanitarios: Formas de gestión. Ediciones Díaz de Santos.

Bonifaz Villar, C. (2012). Desarrollo de habilidades directivas. México: Red Tercer Milenio.

Cruz, F., López, A., y Ruiz, C. (2017) Sistema de gestión IS0 9001-2015: Técnicas y herramientas de ingeniería de calidad para su implementación. Revista Ingeniería, Investigación y Desarrollo, 17 (1), 59-69.

Díaz Figueroa, J., Medina Quintero, J., y de la Garza Ramos, M. (2011). El liderazgo en las empresas para la obtención de ventaja competitiva en pymes turísticas del sur de Tamaulipas. Red Internacional de Investigadores en Competitividad. Memoria del V Congreso, 5(1), 773-750. Recuperado de https://www.riico.net/index.php/riico/ article/view/595/797

Enríquez Castillo, I., y López Ávila, R. M. (2016). Descripción de las características psicosociales de los líderes en empresas jóvenes de la región de $\mathrm{Cd}$. Cuauhtémoc, Chih., Méx. Cultura Científica y Tecnológica, 13(59), 180-191. Recuperado de https:// erevistas.uacj.mx/ojs/index.php/culcyt/article/view/1459

Esquivel, A., León, R., y Castellanos, G. (2017). Mejora continua de los procesos de gestión del conocimiento en instituciones de educación superior ecuatorianas. Retos 
de la Dirección, 11(2), 56-72. Recuperado de http://scielo.sld.cu/pdf/rdir/v11n2/ rdir05217.pdf

Fernández, M. (2017). Liderazgo global ¿Una nueva teoría? Pizarrón Latinoamericano, 8(7), 37-48. Recuperado de http://openjournal.unimet.edu.ve/index.php/Revista Pizarron/article/view/357/331

Franco, M., y Urbano, D. (2010). El éxito de las pymes en Colombia: un estudio de caso en el sector salud. Revista Estudios Gerenciales, 26(114), 77-97.

Fundación Europea para la Gestión de la Calidad. (2018). Modelo EFQM de calidad y excelencia. Recuperado de http://www.efqm.org

Fundación Iberoamericana para la Gestión de la Calidad. (2019). Modelo de la excelencia. Recuperadodehttps://www.fundibeq.org/images/pdf/Modelo_Iberoamericano_V_ 2019_.pdf

García-Cabrera, A., y García-Soto, M. (2016). Calidad del entorno e internacionalización de la pyme: un análisis regional en España. Emprendimiento y Negocios Internacionales, 1(1), 11-18. Recuperado de https://ojsspdc.ulpgc.es/ojs/index. $\mathrm{php} / \mathrm{ENI}$

Gil-Osorio, I. M., e Ibarra-Lopesierra, S. (2014). Incidencia del liderazgo en los factores críticos del éxito como estrategia competitiva empresarial. Revista Dimensión Empresarial, 12(2), 117-126. Recuperado de http://www.scielo.org.co/pdf/diem/ v12n2/v12n2a10.pdf

González, L., Tapia, M., Hernández, D., y Luna, A. (2016). Factores críticos de éxito en la mejora de la calidad de la industria manufacturera en el municipio de Celaya. Pistas Educativas, 38(121), 99-116. Recuperado de http://www.itcelaya.edu.mx/ ojs/index.php/pistas/article/view/506/581

Guerrero, M. (2017). Los estilos de liderazgo en empresarias ecuatorianas y su influencia en el desempeño de sus colaboradores. INNOVA Research Journal, 2(1), 151-161. doi:10.33890/innova.v2.n2.2017.178

Hitt, M.; Ireland, R., y Hoskisson, R. (2015) Administración estratégica. Competitividad y globalización: conceptos y casos. Cengage Learning.

Leonard, D. (2010). The characteristics of quality leadership. ASQ Quality Management Division Conference, Improvements Through People, Processes and Performance.

León, G. E., Marulanda, N., y González, H. (2017). Factores clave de éxito en la implementación de lean manufacturing en algunas empresas con sede en Colombia. Tendencias, 18(1), 85-100. doi:10.22267/rtend.171801.66 
Lezama, M., Cruz, M., y Pico, B. (2015). El liderazgo estratégico moderno y el mejoramiento del desempeño del capital humano. Red Internacional de Investigadores en Competitividad Memoria del IX Congreso, 9(1), 242-261. Recuperado de https://www. riico.net/index.php/riico/article/view/15/15

López Martínez, E. (2013). La importancia del liderazgo en las organizaciones (trabajo final de grado para Diplomatura en Ciencias Empresariales). Universidad Autónoma de Barcelona, Barcelona, España. Recuperado de https://ddd.uab.cat/pub/ tfg/2013/110463/TFG_elopezmartinez.pdf

Luna, J. (2012). Influencia del capital humano para la competitividad de las pymes en el sector manufacturero de Celaya, Guanajuato (tesis de doctorado inédita). Universidad de Celaya, Celaya, México.

Montañez Moya, G. (2015). Hacia el perfil del liderazgo responsable empresarial. Revista Iberoamericana de Contaduría, Economía y Administración, 4(7), 136-148. Recuperado de http://www.ricea.org.mx/index.php/ricea/article/view/44/129

Organización para la Cooperación y Desarrollo Económicos y el Banco de Desarrollo de América Latina (2019). América Latina y el Caribe 2019. Políticas para pymes competitivas en la Alianza del Pacífico y países participantes de América del Sur. https://doi.org/10.1787/60745031-es

Organización para la Cooperación y Desarrollo Económicos y la Comisión Económica para América Latina y el Caribe (2012). Perspectivas Económicas de América Latina 2013: Políticas de pymes para el Cambio Estructural. http://dx.doi.org/10.1787/ leo-2013-es

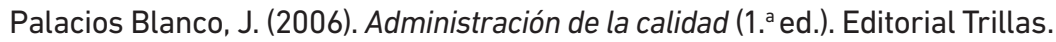

Pedraza, N.A., Lavín, J., Delgado, G., y Bernal, I. (2014). Prácticas de liderazgo en empresas comerciales en Tamaulipas (México). Revista Facultad de Ciencias Económicas: Investigación y Reflexión, 23(1), 251-265. Recuperado de http//www.redalyc.org/ articulo.oa?id=90933063014

Pertúz, F. (2018). Liderazgo transformacional en empresas sociales desde la perspectiva ética de la responsabilidad social empresarial. Telos, 20(2), 377-400. Recuperado de http://ojs.urbe.edu/index.php/telos/article/view/2736/2567

Quintero, P., Álvarez, C., Calderón, G., y Granada, K. (2011). El director como líder creativo. Revista Ad-Minister, 19, 67-81. Recuperado de https://www.redalyc.org/ pdf/3223/322327250004.pdf

Ramseook-Munhurrun, P., Munhurrun, V., y Panchoo, A. (2011) Total quality management adoption in a public hospital: evidence from mauritius. Global Journal of 
Business Research, 5(3), 67-77. Recuperado de https://econpapers.repec.org/ RePEc:ibf:gjbres:v:5:y:2011:i:3:p:67-77

Rougan, D. (2015). A study on TQM Development, performance, and sustenance in service industries through effective communication, critical success factors and market orientation. IOSR Journal of Business and Management (IOSR-JBM), 17(1), 1-12. Recuperado de www.iosrjournals.org

Santamaría Peraza, R. (2017). Factores de calidad determinantes del éxito empresarial en las pymes, aproximación del estado del arte. Ingeniería Industrial: Actualidad y Nuevas Tendencias, 5(19), 105-118. Recuperado de http://www.redalyc.org/ pdf/2150/215055006008.pdf

Schniederjans, M., Mellat, M., Nabavi, M., Subba, S., y Raghu-Nathan, T. (2018). Comparative analysis of Malcolm Baldrige National Quality Award Criteria: an empirical study of India, Mexico, and the United States. Quality Management Journal, 13(4), 7-21. doi:10. 1080/10686967.2006.11918569

Sistema Económico Latinoamericano y del Caribe. (2010). Visión prospectiva de las pequeñas y medianas empresas (pymes). Repuestas ante un futuro complejo y competitivo. Recuperado de http://iberpyme.sela.org/aDocs/VisionprospectivaPYMES.pdf

Spears, L. (2010). Character and servant leadership: ten characteristics of effective, caring leaders. The Journal of Virtues \& Leadership, 1(1), 25-30. Recuperado de https:// www.regent.edu/acad/global/publications/jvl/vol1_iss1/Spears_Final.pdf

Talib, H., Ali, K., e Idris, F. (2013). Quality management framework for the SME's food processing industry in Malaysia. International Food Research Journal, 20(1), 147-164. Recuperado de http://www.ifrj.upm.edu.my/20\%20(01)\%202013/21\%20IFRJ\%20 20\%20(01)\%202013\%20Hayati\%20(040).pdf

Union of Japanese Scientists and Engineers (s. f.) Deming Prize. Recuperado de http://www. juse.or.jp/deming_en/

Wanjiru, L. y Kilika, J. (2016). Firm resources, core competencies and sustainable competitive advantage: an integrative theoretical framework. Journal of Management and Strategy, 7(1), 98-108. doi:10.5430/jms.v7n1p98 
